\title{
ADMINISTRACIÓN PÚBLICA COMPARADA: CONCEPTOS, MÉTODOS Y CAMPO DE INVESTIGACIÓN
}

\author{
COMPARATIVE PUBLIC ADMINISTRATION: CONCEPTS, METHODS \\ AND RESEARCH AREAS
}

\author{
Hellmut Wollmann \\ Humboldt Universität, Berlín / Alemania \\ hellmut.wollmann@rz.hu-berlin.de \\ https://orcid.org/0000-0003-1678-9715
}

Recibido/Received: 22/01/2020

Modificado/Modified: 09/03/2020

Aceptado/Accepted: 20/04/2020

\section{RESUMEN}

Abordando como punto central el tema de la Administración Pública Comparada (APC), el artículo trata de aportar una visión general de su evolución histórica, el contenido de su objeto de estudio, los conceptos y métodos así como los principales campos de investigación y resultados. Se destaca el desarrollo dinámico que APC ha tenido recientemente evidenciado por el aumento significativo de publicaciones (por ejemplo manuales) y de redes relevantes e instituciones dedicadas a la investigación sobre APC. En resumen se argumenta que, por un lado, APC ya ha logrado (en palabras de Christopher Pollitt) una 'vitalidad notable' mientras que, por otro lado, aún quedan problemas importantes, tanto conceptuales como metodológicos, por resolver.

PALABRAS CLAVE

Definición de Administración Pública Comparada (APC); conceptos y diseño de investigación; métodos (cualitativos y cuantitativos); campos de investigación.

\section{SUMARIO}

1. Introducción. 2. Breve desarrollo histórico de la investigación de la Administración Pública Comparada (APC). 3. Diseño y métodos de investigación sobre la APC. 4. Los métodos comparativos. 5. El desarrollo reciente y la situación actual de la investigación de la APC: un breve resumen. 6 . Dinámicas recientes. 7. Resumen y perspectivas. Bibliografía.

\footnotetext{
ABSTRACT

Addressing Comparative Public Administration (CPA) article gives an overview of the history, subject matters, concepts, methods as well as of major research areas and findings It points at the dynamic development CPA has recently experienced as evidenced by the upsurge of thematically relevant publications (such as handbooks) and of pertinent (international) research networks and institutions. In a concluding assessment it is argued that, on the one hand, CPA conclusion it is $t$ is argued that, on the one hand, CPA has attained an (in the words of the late Christopher Pollitt) 'remarkable vitality' while, on the other hand, important conceptual and methodological problems still need to be solved in CPA research.
} 


\section{KEYWORDS}

Definition of Comparative Public Administration (CPA); Concepts and Research Designs; Quantitative and Qualitative Methods; Fields of Research.

\section{CONTENTS}

1. Introduction. 2. Short history of the Comparative Public Administration (CPA) research. 3. Research design and methodology. 4. Comparative methods. 5. Recent development and current state of CPA research. 6. Recent dynamics. 7. Summarizing and perspectives. References

\section{INTRODUCCIÓN}

Comprender y definir el concepto de 'administración pública' se ha erigido como un esfuerzo permanente dentro de los debates académicos que tienen por objeto el estudio de las administraciones públicas, particularmente en lo que respecta a su estudio comparado (Comparative Public Administration).

En apariencia, ha existido un inicial consenso académico en el que la triada de organización, personal y comportamiento serviría suficientemente para capturar los componentes claves de lo que constituye la administración pública (AP). Tanto es así que las investigaciones iniciales en ámbito de la administración pública comparada (APC) se focalizaban en su vertiente organizativa, como por ejemplo la metáfora de la "máquina burocrática del gobierno' de Peters (1978), la gestión de los recursos humanos (los denominados 'sistemas de servicio civil' por Bekke et al. $(1999,2000)$ y al comportamiento administrativo que se ocupaba del estudio de las actitudes de las élites administrativas (Aberbach et al. 1981).

De manera que para diseccionar analíticamente la administración pública en sus diversas funciones operativas puede servir el famoso POSDCORB acuñado por Gulick (acrónimo, en sus siglas en inglés, de Planificar, Organizar, Crear equipos de asesoramiento y estudio, Dirigir, Coordinar, Informar y Presupuestar) que abre el camino para el estudio por separado de las diferentes unidades funcionales dentro de la AP. Este desarrollo inicial ha sido adoptado por los más recientes enfoques de la Nueva Gestión Pública (New Public Management) en adelante: NGP, que ha inspirado su interés por el diseño de investigaciones de APC encaminadas al estudio de los ámbitos administrativos encargados de, por ejemplo, la gestión del desempeño, el control o la evaluación.

Otra propuesta analítica para el estudio de la AP consiste en diferenciar áreas políticas específicas, por ejemplo, la administración de asuntos o políticas sociales, ambientales, etc.

Más recientemente, se han producido cambios importantes en estos enfoques iniciales derivados del impacto de las políticas neoliberales que han justificado la implantación de la NGP y, sobre todo, la institucionalización de unas nuevas interacciones entre los sectores público y privado y el tercer sector, que han significado un importante cambio de paradigma, ampliándolo, en el enfoque la investigación de la APC, (Wollmann, 2018).

En primer lugar, la hegemonía primigenia del sector público en la realización de funciones públicas ha sido sustituida por una disgregación institucional que lleva implícita la pluralización y privatización de la acción pública. Asimismo, y en palabras de Peters, "la distinción entre lo que es público y lo que es privado se ha vuelto difusa" (Peters, 2010, 3). En este contexto, han proliferado términos acuñados para captar esta nueva realidad tales como los de agencificación (Verhoest et al. 2012) y corporatización (Grossi y Reichard 2018) de funciones públicas, la conformación de organizaciones híbridas público-privadas, 
mediante partenariados o PPP's, (Vecchi y Hellowell, 2018) así como procesos de 'remuncipalización' (Wollmann, 2016: 323 y ss.) que han abierto nuevas líneas de investigación en el marco de la APC.

De modo similar, las relaciones entre la administración pública y la sociedad civil han sido objeto prioritario de investigaciones dentro del ámbito de la APC (Pierre, 1995; Brans, 2012; Page, 2003: 421). Además, como consecuencia de los impactos derivados de las recientes políticas de austeridad fiscal que han conllevado una retirada de los poderes públicos de la prestación de servicios públicos, las organizaciones y actores encuadrados dentro del denominado Tercer Sector (Salamon y Sokolowski, 2016) han jugado progresivamente cada vez un mayor papel en este campo y, en consecuencia,) a atraer una creciente atención en la investigación de APC (Wollmann, 2016: 324 y ss).

Finalmente, se hace preciso mencionar la coincidencia conceptual y empírica que la investigación de APC tiene con otras variantes de investigación tales como el enfoque de las políticas públicas o policy research (Van de Walle y Brans, 2017: 105). Aplicando el concepto heurístico del 'ciclo de las políticas' o "policy cycle”, (Jann y Wegrich, 2007) en el que se distinguen las fases de la formulación de las políticas públicas, su implantación y su terminación/evaluación, se pone de manifiesto que la administración pública está estrechamente imbricada y conectada con todas las tres fases y ello se hace particularmente evidente en la fase de implantación donde la administración pública ejerce como actor clave. Esto también es cierto con respecto a las instituciones y actores conectados con la fase de la formulación de políticas, así como con la del fin del 'ciclo', es decir, de la evaluación de la política (y del "feedback" de las informaciones y datos que proveen las evaluaciones, además de los procesos de aprendizaje que conllevan o policy learning). Por todo ello, las investigaciones sobre APC se encuentran íntimamente ligadas a los trabajos de investigación sobre la implementación de las políticas públicas (implementation research, Pressman y Wildavsky, 1973) así como con las investigaciones sobre la evaluación de políticas públicas (Wollmann, 2003; Pattyn et al. 2018) y aprendizaje político (policy learning, (Dunlop y Radaelli, 2018).

\section{BREVE DESARROLLO HISTÓRICO DE LA INVESTIGACIÓN DE APC}

Una comprensión cabal de la APC sugiere realizar previamente, como ocurre en la mayoría de conceptos sociales, una breve síntesis del desarrollo de la APC misma sustentada en los trabajos de, entre otros, Farazmand (1996), Pollitt (2011:116 y ss.), Raadschelders y Vigoda-Gadot (2014: 446 y ss.) y Kuhlmann \& Wollmann (2019: 6 y ss.).

Desde una perspectiva histórica, la dedicación académica a la AP puede remontarse al siglo dieciocho en Alemania cuando el Rey de Prusia se hizo eco de los planteamientos académicos en torno al Cameralismo (Kameralwissenschaften) en las, por entonces, recién instauradas universidades (en 1723 se crearon las de Halle y Frankfurt/Oder). La intencionalidad era la formación y consolidación de una nueva clase de administradores públicos versados en el, entonces todavía rudimentario, conocimiento científico que tuviera aplicabilidad en los ámbitos de la agricultura y producción manufacturera propios del modelo de Estado mercantilista. Estas premisas fueron posteriormente desarrolladas, ya en el siglo diecinueve, en la que se denominó 'Ciencias del Estado' (Staatswissenschaften) (Kickert y Stilman, 1996: 66), cuyo representante más prominente fue Lorenz von Stein (1815-1890) quien definió a la administración pública como el 'estado en acción' (arbeitender Staat). El postuló que el estudio y la enseñanza de la administración pública 
debiera constituirse como una 'ciencia integradora', incluyendo el derecho público, la sociología y las finanzas públicas. De manera que este pensador puede considerarse como uno de los precursores de la Ciencia de la Administración en Alemania (Sager et al., 2018: 101). Sin embargo, hacia finales del siglo diecinueve el interés académico por la administración pública estuvo inspirado por la hegemonía del positivismo jurídico, dejándose a un lado las perspectivas interdisciplinarias de carácter más empírico de los Staatswissenschaften referidos anteriormente.

Aunque a mediados del siglo diecinueve numerosos estudiantes norteamericanos siguieron cursos en universidades alemanas, principalmente en Halle, Heidelberg y Berlín) $\mathrm{y}$, en consecuencia se familiarizaron con la tradición alemana de los Staatswissenschaften. Entre ellos cabe destacar a Herbert Adams y John Burgess, quienes a su vez fueron profesores de Woodrow Wilson y Frank Goodnow (Sager et al. 2018: 31 y ss.). Y así fue como, de este modo, los Staatswissenschaften influyeron en el estudio académico emergente de la AP en los Estados Unidos.

En ese país, Woodrow Wilson (1856-1924), quien en 1887 escribió su artículo pionero 'El Estudio de la Administración' (The Study of Administration, Wilson, 1887) puede considerarse el fundador intelectual de la AP como disciplina académica. Para ello, propuso cuatro conceptos fundamentales: primero, la separación entre política y administración; segundo, el análisis comparativo (!) entre organizaciones públicas y privadas, afirmando que "en ningún aspecto del campo de los estudios políticos se podría utilizar el método históricocomparativo, con más seguridad que en esta área de la administración"); tercero, mejorar la eficiencia adoptando prácticas y comportamientos de tipo empresarial; y, finalmente, mejorar la eficacia y eficiencia de los servicios públicos mediante la gestión (management) y la formación de empleados públicos, introduciendo, a la vez criterios de mérito y capacidad para su valoración. El debate académico posterior en torno al objeto de estudio de la AP se focalizó en la cuestión de la separación entre Política y Administración, lo que fomentó una concepción de la AP como un subsistema cerrado regido por "principios' (principles) internos e 'inmune' a las influencias externas. Paralelamente, la perspectiva comparativa decayó hasta límites en los que fue ignorada.

Sólo con posterioridad a la Segunda Guerra Mundial, la CPA surgió como una disciplina manifiestamente comparativa y propulsada por un enfoque centrado en el cambio administrativo (development administration). Esta orientación comparativa fue fomentada desde la Fundación Ford cuyos programas se dirigían a ayudar a países en desarrollo durante el proceso de descolonización. Tal es así, que en 1954, se fundó el 'Grupo de Administración Comparada' (Comparative Administration Group, CAG), bajo el liderazgo de Fred Rigg, dentro de la American Society for Public Administration. El CAG actuó como fuerza propulsora en el desarrollo de la de CPA, bajo el impulso de Fred Riggs (1917-2008) y Ferrel Heady. En 1966 Heady publicó su libro pionero Administración pública. Una perspectiva comparada (Public Administration. A Comparative Perspective) el cual alcanzó su sexta edición en 2001. Aunque con una orientación práctica y modernizadora (developmental) Riggs defiende una investigación de APC que sea "empírica, nomotética y ecológica' y que aspire a formular y probar hipótesis que permita la construcción de una teoría comprensiva (grand theory) de la AP (Riggs, 1962: 116).

Con posterioridad y durante la década de los setenta del siglo pasado, se produjo un declive de las investigaciones comparativistas sobre la AP no sólo debido a la falta de financiación por parte de la Fundación Ford que las propició, sino, también por el progresivo desinterés de las agencias gubernamentales. Además "el desencanto más grave venía de la promesas científicas no cumplidas o del fracaso de producir una teoría general de los 
sistemas administrativos” (Brans, 2003: 427; Farazmand, 1996: 343).

Hacia finales de la citada década, destacan dos publicaciones que contemplaban como tema nuclear a la burocracia como el objeto de estudio más importante de la investigación sobre APC. En 1978 Peters publicó su libro La Política de la Burocracia (Politics of Bureaucracy) el cual llegó a su sexta edición, con el añadido del subtitulo 'Introducción a Administración Pública Comparada'). Más tarde, en 1981, Aberbach y colaboradores publicaron su obra comparativa sobre burócratas y políticos en las democracias occidentales, Bureaucrats and Politicians in Western Democracies. Este trabajo resultaba influido por el paradigma behaviorista dominante en las ciencias sociales de la década de los 70 y utilizaba además un enfoque cuantitativo para medir y dar cuenta del comportamiento de las élites político-administrativas.

A principios de la década de los noventa se produjo un auge y aceleración de los estudios comparativos (Pollitt, 2011: 118 y ss.). Este auge implico también el surgimiento de una especialización en la investigación ampliando el campo de estudio. En este contexto merecen destacarse tres factores que actuaron como propulsores del desarrollo de la APC.

En primer lugar, varias oleadas de reformas en el sector público tuvieron un impacto significativo en la agenda de la APC. En los años ochenta un conjunto de actores internacionales propusieron la transformación masiva del sector público bajo el mantra de la "reinvención del gobierno" (reinventing government) que tuvo su origen en Estados Unidos y de la 'Nueva Gestión Pública', New Public Management, que después de surgir en el Reino Unido tuvo una importante acogida en otros países y que ha inspirado numerosas investigaciones y publicaciones sobre APC (Pollitt y Bouckaert, 2017; Kuhlmann y Wollmann, 2019).

En segundo lugar, a partir de los años 90, la implosión y transformación de las estructuras político-administrativas en los países excomunistas de la Europa del Este ha propiciado nuevas oleadas de investigaciones.

Tercero, se hace preciso mencionar, en este contexto, la creación del Grupo Europeo de Administración Pública (European Group for Public Administration (EGPA) que fue fundado en 1975 en el marco del Instituto Internacional de Ciencias Administrativas (International Institute of Administrative Sciences). El EGPA ha jugado un papel crucial en el desarrollo y la expansión de APC en Europa y fuera de ella (Pollitt, 2011: 123).

\section{DISEÑO Y MÉTODOS DE INVESTIGACIÓN SOBRE LA APC}

En términos generales, las investigaciones sobre APC están dirigidas al análisis de los fenómenos administrativos y a identificar sus factores causales. Por lo tanto, como cada proyecto de investigación en ciencias sociales, tal investigación se enfrenta a un triple desafío. Primero se hace preciso delimitar y definir el objeto de estudio, es decir, el "qué" o, en términos metodológicos, la "variable dependiente" o explanandum. En segundo lugar, hay que identificar los factores que (causalmente) determinan la "variable dependiente", es decir, el "por qué" y la "variable independiente" o explanans. Además hay que escoger el método idóneo que permita identificar ("explicar") las relaciones causales entre las variables "dependientes" e "independientes" pertinentes.

\subsection{Variables dependientes (explanandum)}

En vista de la complejidad multifacética de la realidad administrativa, la primera tarea crucial consiste en delimitar y definir de modo analítico segmentos o bloques empíricos que 
puedan operacionalizarse (analytical building blocks véase Aberbach y Rockman, 1988; Peters, 1988; Pierre, 1995; Brans, 2003: 431). Por ejemplo Peters (1988) propuso cuatro "bloques empíricos"; empleo público, organizaciones públicas, comportamiento burocrático y relaciones político-administrativas. Por otro lado, Pierre 1995 sugirió tres 'bloques': dinámicas de las organizaciones burocráticas, relaciones político-administrativas y relaciones entre la administración y la sociedad civil. Aunque este concepto sea de utilidad para analíticamente delimitar la complejidad del estudio de la administración pública los esquemas propuestos por Peters y Pierre revelan la incertidumbre e incluso la arbitrariedad en la definición de las unidades de análisis (analytical units). De manera significativa, Pierre reconoce que "analíticamente no disponemos de definiciones útiles para alguno de los fenómenos nucleares en el estudio de la administración pública" (Pierre, 1995: 6).

Con este trasfondo, cada proyecto de investigación sobre APC se encuentra, inicialmente, ante el desafío crucial de definir de forma adecuada su 'unidad de análisis' y sus 'variables dependientes'.

\subsection{Variables independientes (explanans)}

Como en el resto de las ciencias exactas, también en las ciencias sociales la selección de las variables independientes o explicativas está sujeta a su delimitación a partir de las hipótesis que permitan el establecimiento de relaciones de causa-efecto.

Sin embargo, en ausencia de formulaciones teóricas suficientemente fundamentadas en evidencias empíricas, el conocimiento sobre relaciones causales en el ámbito de la APC debe en muchos casos valerse de estrategias heurísticas que permitan guiar estudios de carácter exploratorio al objeto de explicar tentativamente las hipótesis planteadas.

Dentro de las aproximaciones (approaches) exploratorias derivadas de los procedimientos de tipo heurístico para satisfacer explicaciones que permitan dar cuenta de la realidad administrativa, se pueden identificar varias corrientes o escuelas que han arraigado en el debate científico.

En primer lugar, se puede identificar una concepción inicial en los debates que se ocupan de las diversas variantes del neo-institucionalismo (neo-institutionalism, Peters, 2011; Kuhlmann y Wollmann, 2019: 44 y ss.). La variante de esta corriente que se focaliza en los actores (actor-centred, Scharpf, 1997) pone de relieve la influencia que los actores políticos y económicos ejercen en el desarrollo institucional. Por otra parte, el institucionalismo histórico subraya la influencia que las estructuras institucionales, tanto vigentes como tradicionales, ejercen sobre el desarrollo institucional hasta el punto de crear "dependencias del sendero" (path dependencies, Pierson 2000). También, según el institucionalismo sociológico el desarrollo institucional resulta modulado por los aspectos cognitivos y socioculturales inherentes a los actores que son trasladados a las instituciones. Finalmente, el institucionalismo discursivo (discursive) pone el énfasis en los discursos y las ideas dominantes que producen impactos en el desarrollo institucional (véase Schmidt, 2008). De igual manera, el isomorfismo normativo (normative ismorphism) hace hincapié en la influencia de las ideas y de los conceptos (DiMaggio y Powell, 1991).

A todo lo anterior habría que añadir la cultura política y social que han sido señaladas como importantes factores explicativos (Peters, 2010) con las limitaciones que menciona este autor en el sentido de que 'la cultura política es un concepto difícilmente delimitable y su medición aún más" (Ibid., 35).

En esa misma línea, las aproximaciones ecológicas como un complejo contextual han sido puestas de relieve como variables de influencia por Caiden y Caiden (1990), quienes siguen a Riggs (1962) como el primer autor que puso el foco en el aspecto ecológico. 
Ni que decir tiene que los procesos de internacionalización y globalización y su influencia en la soberanía de los Estados han sido fenómenos determinantes en el desarrollo y evolución de las estructuras y procesos político-administrativos (Farazmand, 1999; Chandler, 2014).

\section{LOS MÉTODOS COMPARATIVOS}

Dentro del marco de las ciencias sociales, la investigación sobre APC dispone de dos caminos metodológicos (approaches) para identificar relaciones de causalidad: los diseños cuantitativo-estadísticos basados en datos agregados y encuestas, por un lado, y, por otro, el diseño cualitativo basado mayoritariamente en estudios de caso. Generalmente, (Lijphart, 1975: 686), la aplicabilidad de la aproximación cuantitativa se sustenta en la consideración de "pocas variables y muchos casos $(\mathrm{N})$ ), ( few variables many cases, large $N$ ) mientras que el enfoque cualitativo está basado en considerar "muchas variables y pocos casos, N pequeño' (many variables, few cases, small $N$ ).

Respecto a la aproximación cuantitativa-estadística, su utilización ha sido limitada tanto en los estudios de carácter genérico sobre la administración pública como en los más específicos sobre estudios comparativos. En concreto, ha sido utilizado en investigaciones acerca de los comportamientos y valores, entre otros, de los actores administrativos y estaban basados en la aplicación de encuestas (Aberbach et al., 1981). Aunque, más recientemente, el uso de métodos sustentados en una aproximación cuantitativo-estadística ha cobrado relevancia en estudios de comparaciones internacionales a gran escala (Verhoest et al., 2012; Hammerschmid et al., 2016).

Con todo, es la aproximación cualitativa, sustentada en estudios de caso (case studies) la que ha prevalecido en los trabajos de investigación de carácter comparativo en sus diversas variantes. Así, una primera propuesta es la realizada por Przeworski y Teune (1970), y posteriormente por Lijphart (1975) está basada en la lógica (de alguna manera: cuasiexperimental) de que los casos elegidos deben ser lo más semejantes posibles (si no iguales, (ceteris paribus) de manera que las variables pertinentes, susceptibles de expresar la causalidad, se encuentren (en términos metodológicos) 'controladas'. A este respecto, Raadschelders y Vigoda-Gadot (2015: 460 y ss.) amplían esta estrategia de investigación refiriéndose a dos opciones: la elección de casos más similares, (most similar systems design, MSSD), o de casos más diferentes (most different systems design, MDSD). Pero, aunque esta lógica cuasi-experimental presente fortalezas desde un punto de vista teórico, en la práctica es a menudo prácticamente imposible crear situaciones ceteris paribus que serían imprescindibles para una aplicación rigorosa de la lógica cuasi-experimental (Pollitt, 2011: 121).

De todas maneras, las investigaciones comparativas descansan en una estrategia de estudios de casos múltiples (multi cases), Eckstein (1975). Sustentada en el estudio de casos (y los típicos instrumentos de recogida de datos: entrevistas, observaciones, análisis documental, entre otros) esta estrategia facilita la realización de descripciones y análisis de carácter "grueso" (thick description, Raadschelders, 2011: 831 y ss.; Kuhlmann y Wollmann, 2019: 6), si bien es cierto que la indagación cualitativa, basada en los estudios de caso, pude conducir a situaciones casuales (serendipia) o serendipity, Merton (1957: 103 y ss.), es decir, a 'hallazgos inesperados' que pueden evocar nuevas hipótesis o modificar las ya existentes. En cualquier caso, es de sobra conocido que los estudios de caso único, aunque proporcionan evidencias y explicaciones ad hoc, (ad hoc explanations), son 
difícilmente generalizables más allá del propio caso. Por el contrario, una estrategia de casos múltiples se considera dispone de potencial analítico suficiente para generar explicaciones y generalizaciones que pueden contribuir a la construcción de teorías de medio alcance (middle range theories).

Sin embargo, el potencial analítico de una estrategia de casos múltiple depende de la selección de los mismos mediante una selección sesgada que tiene en cuenta el propósito de la investigación en sus varios aspectos, (purposive sampling y purposeful selection) de los casos u objetos de estudio (países, etc.), o de la 'selección apuntada' de los países (o casos etc.) (Fitzpatrick et al. 2011: 823; van de Walle and Brans, 2018: 110). Así, en contraste con la mera compilación de países (o casos) por razones de conveniencia o de practicabilidad (fácil acceso, conocimiento de lenguas etc.), en la búsqueda de relaciones de causalidad, la estrategia de selección según los objetivos de la investigación, se acerca bastante a la lógica cuasi-experimental.

Además de las tipologías que agrupan países o casos en razón de determinados rasgos y criterios (Jreisat, 2005: 232; Kuhlmann y Wollmann, 2019; Raadschelders y Vigoda-Gadot, 2015: 432 y ss.) es posible, como una variante de selección antes apuntada, delimitar el estudio de países en función de tipologías sustentadas en características de tradiciones históricas (por ejemplo, Painter y Peters, 2010, distinguiendo y escalando países "Napoleónicos" y "Germánicos", además de Kuhlmann y Wollmann (2019) diferenciando países en función de su localización geográfica, por ejemplo, Europa Continental y de Europa Centro-Este) . Tales tipologías deben permitir explorar si a lo largo de su desarrollo pueden identificarse diferencias significativas que permitan la realización de inferencias de tipo causal.

\section{EL DESARROLLO RECIENTE Y LA SITUACIÓN ACTUAL DE LA INVESTIGACIÓN DE APC: UN BREVE RESUMEN}

Este resumen se basa en un reciente trabajo de Fitzpatrick et al. (2011) en el que se realiza una meta-análisis de 151 artículos publicados entre los años 200 y 2009. También se basa en un artículo de Pollit (2012) de un trabajo similar en el que se reseñan publicaciones de las últimas tres décadas.

Existen trabajos pioneros que se ocuparon del abordaje de la organización burocrática de la administración pública, tales como la monografía de Peters (1978), ya en su sexta edición en 20110, y la monografía editada por Rowat (1988) que se ocupaba de la AP en los "países desarrollados". Otro trabajo iniciático fue el de Aberbach y colaboradores (1981) focalizado en el estudio de las élites burocráticas en las democracias occidentales y que tuvo su continuación en los trabajos de Pierre (1995) centrado en el estudio de las relaciones entre políticos y burócratas en Francia, Alemania, Reino Unido, Japón, Suecia, Kenia y Tanzania. En esta misma línea se encuentra también el trabajo de Page y Wright (1999) centrado en el estudio de las élites burocráticas en los países de la Europa occidental. Estos estudios ponen de manifiesto una perspectiva etnocéntrica en tanto que el estudio se concentró en los países desarrollados con algunas excepciones como los trabajos editados por Dwivedi y Henderson (1990) sobre el estudio de la AP en una perspectiva global, así como los de Farazmand (1991) con su manual sobre Administración Pública Comparativa y de Desarrollo.

Más adelante, con la llegada del nuevo siglo, los estudios de investigación desde una perspectiva comparada se interesaron por el empleo y el servicio público, que conllevaron la realización de estudios variados con un alcance regional. Estos estudios incluían países de 
Europa Occidental (Bekke y van der Meer 1999; 2000), 27 países europeos (Demmke y Moilanen, 2013) países de Europa Central Y Oriental (Verheijen, 1999), países AngloAmericanos (Halligan, 2004) así como países asiáticos (Burns y Bowornwathana, 2001).

Aunque previamente, desde la última década del siglo pasado, despuntó otra corriente de investigación con un enfoque de APC relacionada con la modernización del sector público y que fue inspirada por la Nueva Gestión Pública (New Public Management, NPM), sobre todo en países de la OCDE. Esta corriente fue propulsada en parte por iniciativas y financiación la propia OCDE, del Banco Mundial (World Bank) y la Unión Europea apoyando proyectos de investigación que tenían por objeto de estudio los gobiernos centrales y reformas sectoriales. Entre ellos se encuentran los de Peters y colaboradores (2000) sobre el 'ejecutivo núcleo'. core executive en 12 países); Peters y colaboradores (2006) (sobre 'asesorías de gobierno', advice to government en 8 países europeos); Verhoest y colaboradores (2012) (sobre 'agencias públicas' en 29 países, sobre todo de la Unión Europea), así como el de y Hammerschmidt et al. (2016) sobre reformas del sector público en 17 países europeos.

Más recientemente se han llevado a efecto investigaciones centradas en los niveles subnacionales y/o locales a partir del impulso de programas financiados por la Unión Europea 'reformas de sector público local'. A partir de la formación de un consorcio internacional de investigadores se generaron una serie de publicaciones, por ejemplo Kuhlmann y Bouckaert (2016), Wollmann et al. (2016) que se ocupan de la prestación de servicios públicos en países de la Union Europea), Grossi y Reichard (2016), sobre la 'corporatización' en el nivel local), Lippi et al. (2019), sobre la prestación de servicios públicos en países de Europa del sur. Merecen también mencionarse los 'informes globales' encargados y publicados por 'Ciudades Unidas y Gobierno Local' (“United Cities and Local Government”, UCLG). Con una cobertura global mediante la clasificación de los países en 'regiones' (por ejemplo Europa, América de Norte, Asia del Pacifico etc.) el 'informe global' provee también datos importantes sobre los sistemas administrativos sub-nacionales/locales (véase UCLG 2008).

Por lo demás, las transformaciones emergentes en los países de la Europa Central y Oriental (CEE) a partir de 1990 tuvo como consecuencia el surgimiento de una oleada de investigaciones fomentadas desde el Banco Mundial et de la Unión Europea. En gran parte fueron dirigidas al nivel central, véase, por ejemplo, Verheijen y Coombes (1998) sobre 'innovación en gestión pública. Perspectivas desde el Este y el Oeste'), Nunberg et al. (1999) sobre la 'transición administrativa en los países de Europa Central y Oriental'), Verheijen (1999). Sobre los 'sistemas de servicio civil en Europa Central y Oriental') así como, los trabajos apoyados en estudios de casos de Dimitrov et al. (2006) sobre 'instituciones de gobierno central' en Hungría, Polonia, Chequia, Bulgaria. Respecto al nivel local, se pueden consultar también los capítulos relevantes en las publicaciones de Wollmann et al., 2016 y Kuhlmann y Bouckaert (2016). En este ámbito, el análisis de la gestión, en la que confluyen aspectos tanto institucionales como procedimentales, puede contribuir notablemente a la investigación comparada, como se pone de manifiesto en los trabajos de Lankina et al. (2006) sobre la gestión administrativa en los gobiernos locales de Polonia, Chequia, Hungría y Rusia, así como en los de Kopric et al. (2018).

\section{DINÁMICAS RECIENTES}

Las dinámicas recientes en el desarrollo de la APC se manifiestan en la frecuencia y expansión de ediciones y re-ediciones de manuales y otras publicaciones que estudian el 
fenómeno. Así, por ejemplo, la monografía clásica de Peters sobre política y burocracia (The politics of bureaucracy) que surgió a la luz en 1978 ha alcanzado ya su sexta edición en 2010. Por su parte, en 2014, Kuhlmann y Wollmann publicaron una 'Introducción a la APC' (Introduction to Comparative Public Administration) que en 2019 va por su segunda edición. También en 2015 Raadschelders y Vigoda-Gadot publicaron una monografía sobre la Dimensión Global de Administración Pública y el Gobierno (Global Dimensions of Public Administration and Governance). El manual Handbook of Public Administration, editado por Peters y Pierre en 2003 fue revisado en una segunda edición en 2011. En 2018 apareció un gigantesco trabajo en dos volúmenes Handbook of Public Administration and Management in Europe, editado por Ongaro y van Thiel y que contiene amplias referencias a la APC. Finalmente, debe mencionarse la Encyclopedia of Public Administration preparada por Peters y Thynne. En conjunto, podría afirmarse sin duda que la APC ha alcanzado una creciente atención y visibilidad internacional.

\section{RESUMEN Y PERSPECTIVAS}

A partir de todo lo anterior, cabría colegir que en su desarrollo y perspectivas los enfoques centrados en la APC presentan una situación ambigua. De una parte, a la luz de la compleja y multifacética realidad administrativa, hasta ahora, esta perspectiva solo ha sido capaz de delimitar y analizar aspectos dispersos de esa realidad. Así, no resulta sorprendente que tales investigaciones se hayan reducido a los aspectos micro y meso (middle range), incapaces, por tanto de proveer de las teorías comprensivas (grand theory) intuidas por sus pioneros (Jreisat 1975; Peters 1988 y Brans 2003: 427). Lo que si permanece es una incertidumbre respecto a los métodos de análisis más idóneos para realizar estudios comparados, particularmente en lo que concierne a las técnicas de selección de objetos a comparar (sampling) lo que se constituye en "uno de los problemas más cruciales para la investigación comparada' (Fitzpatrick et al. 2011: 826). A ello habría de añadirse los múltiples retos de carácter no solo conceptual, sino también, teóricos y metodológicos derivados de la perspectiva interdisciplinaria que, a menudo, la investigación sobre la APC, ha adoptado (Raadschelders y Vigoda-Gadot, 2015: 443; Pollitt, 2011: 115, Kuhlmann y Wollmann, 2019: 7). El recurso a varias disciplinas de las ciencias sociales y jurídicas (ciencia política, sociología, derecho, economía etc.) implica echar mano de distintos enfoques (approaches) y métodos particulares más apropiados a cada disciplina. Por lo que, "parece existir una escasa convergencia acerca de una teoría o método específico" (Pollitt, 2011: 114).

Por otro lado, es innegable que, en los últimos años la investigación sobre APC ha hecho significativos avances hacia una consolidación disciplinaria e interdisciplinaria que también queda reflejada en su productividad. Este desarrollo se ha sustentado en un importante crecimiento logístico en términos de asociaciones profesionales y académicas de rango tanto nacional como internacional, así como de redes de investigación, nacionales e internacionales, con sus correspondientes múltiples vías de publicación, recursos financieros y bases de datos, entre otros. En un contexto donde también se ha producido una implosión de ediciones y re-ediciones de manuales y libros que adoptan esta perspectiva. Por ello, a pesar de las limitaciones conceptuales, teóricas y metodológicas, así como de la delimitación fragmentada del complejo objeto de estudio que son la AP, se ha generado un creciente "corpus" de conocimientos y de experiencia investigadora en la que pueden fundamentarse no sólo futuros esfuerzos empíricos, sino también futuros avances teóricos.. Por todo ello no 
es aventurado afirmar que la APC ha ganado una 'notable vitalidad' (Pollitt, 2011: 114) convirtiéndose en una "corriente principal y competitiva" (Pollitt, 2010: 763; Kuhlmann y Wollmann, 2019: 6).

\section{BIBLIOGRAFÍA}

Aberbach J.D. y Rockman B.A. (1988). Problems of Cross-National Comparison”, en Rowat, D.C. (Ed.) Public Administration in Developed Democracies, A Comparative Study. New York: M. Dekker, pp, 419-439.

Aberbach, J. D.; Putnam, R. D. y Rockman, B. A. (1981). Bureaucrats and Politicians in Western Democracies. Cambridge: Harvard University Press.

Baldersheim, H.; Illner, M. y Wollmann, H. (Eds.) (2003). Local Democracies in post-communist Europe. Opladen: Leske + Budrich.

Bekke, H.A. y van der Meer, F. (Eds.) (2000). Civil Service Systems in Western Europe Cheltenham: Edward Elgar.

Bekke, H.A.; Perry, J. y Thoonen, T. (Eds.) (1999). Civil Service Systems in Comparative Perspective. Indianapolis: Indiana University Press.

Brans, M. (2012). "Comparative public administration: From general theory to general frameworks", en: B. G. Peters y J. Pierre (Eds.) The Sage Handbook of Public Administration. London: Sage, pp. 424-438.

Brans, M. y Pattyn, V. (2017). "Validating methods for comparing public policy: Perspectives from academics and "pracademics". Introduction to the Special Issue, Journal of Comparative Policy Analysis, 19(4): 303-312.

Burns,J.P. y Bowornwathana, B. (Eds.) (2001). Civil Service Systems in Asia. New York: Edward Elgar.

Caiden, G. y Caiden, N. (1990). « Towards the Future of Comparative Public Administration”, en: O.P. Dwivedi y K.M. Henderson (Eds.) Public Administration in World Perspective, Ames, Iowa: Iowa State University Press, pp. 363-399.

Chandler, J.A. (Ed.) (2014). Comparative Public Administration. New York: Routledge

Dahl, R. A. (1947). "The Science of Public Administration. Three Problems", en Public Administration Review, 7 (1): 1-11

Demmke, C. y Moilanen, T. (2013). Government transformation and the future of public employment. The impact of restructuring on status development on the central administration of the EU-27, Frankfurt: Peter Lang.

Derlien, H. U. (1992). "Observations on the State of Comparative Administration Research in Europe: Rather Comparable than Comparative", en Governance, 5(3): 279-311, https://doi.org/10.1111/j.1468-0491.1992.tb00041.x

DiMaggio, P.J. y W.W. Powell (1991). The New Institutionalism in Organizational Analysis, Chicago, IL: University of Chicago Press.

Dimitrov, V.; Goetz, K. y Wollmann, H. (2006). Governing after Communism Institutions and Policy Making, Lanham, MD.: Rowman \& Littlefield.

Dunlop, C.A. y Radaelli, C. J. (2018). "Policy Learning and Organizational Capacity" en; E. Ongaro y S. van Thiel (Eds.) The Palgrave Handbook of Public Administration and Management in Europe, Vol. 1, Basingstoke: Palgrave Macmillan, pp. 595-620.

Dwivedi, O.P. y Henderson, K.M. (Eds.) (1990). Public Administration in World Perspective, Ames, Iowa: Iowa State University Press.

Eckstein, H. (1975). Case Study and Theory in Political Science, in: Greenstein, F.E./Polsby, N.W. (eds.) 1975, Handbook of Political Science, Vol. 7. Reading Mass., pp. 100-129.

Farazmand, A. (1991). Handbook of Comparative and Development Public Administration, New York: Marcel Dekker AG.

Farazmand, A. (1996). "Development and Comparative Public Administration, Past, Present and 
Future”, en Public Administration Quarterly, 20 (3): 343-364.

Farazmand, A. (1999). "Globalization and Public Administration", en Public Administration Revierw, 59 (6): 509-522.

Fitzpatrick, J.; Goggin, M.; Heikkila, T.; Klingner, D.; Machado, J. y Martell, C. (2011). "A new look at comparative public administration: Trends in research and an agenda for the future", en Public Administration Review, 71(6): 821-830.

Grossi, G. y Reichard, C. (2016). "Variance in the Institutions of Local Utility Services, Evidence from Several European Countries", en: H. Wollmann, I. Kopric y G. Marcou (Eds.) Public and Social Services in Europe. From Public and Municipal to Private Sector Provision, London: Palgrave Macmillan, pp. 297-312.

Halligan, J. (Ed.) (2004). Civil Service Systems in Anglo-American Countries. London: Edgar Elgar.

Hammerschmid, G.; Van de Walle, S.; Andrews, R. y Bezes, P. (Eds.) (2016). Public Administration Reforms in Europe. The View from the Top. Cheltenham: Edward Elgar.

Heady, F. (2001). Public Administration. A Comparative Perspective. New York: Marcel Dekker.

Jacob, S.; Speer S. y Furubo, J-E. (2015). The institutionalization of evaluation matters: Updating the International Atlas of Evaluation 10 years later, California: SAGE.

Jann, W. y Wegrich, K. (2007). "Theories of the policy cycle”, en: F. Fischer, G. J. Miller and M. S. Sidney (Eds.) Handbook of Public Policy Analysis: Theory, Politics and Methods (Boca Raton: CRC Press), pp. 43-62.

Jreisat, J. E. (2005). "Comparative public administration is back in, prudently". Comparative Public Administration, 65(2): 231-242.

Jreisat, J.E. (1975). "Synthesis and Relevance in Comparative Public Administration", en Public Administration Review. 35 (6): 663-71.

Kickert, W. y Stilman, R. (1996). "Introduction: Changing European States , Changing Public Administration", en Public Administration Review, 56 (1): 65-67.

Kopric, I.; Wollmann, H. y Marcou, G. (Eds.) (2016). Evaluating Reforms of Local Public and Social Services in Europe. London: Palgrave Macmillan.

Kuhlmann, S. y G. Bouckaert (Eds.) (2016). Local Public Sector Reforms in Times of Crisis: National Trajectories and International Comparisons. London: Palgrave Macmillan.

Kuhlmann, S. y Wollmann, H. (2019). Introduction to Comparative Public Administration: administrative Systems and Reforms in Europe. Cheltenham: Edward Elgar Publishing.

Lankina, T.; Hudalla, A. y Wollmann, H. (2008). Local Governance in Central and Eastern Europe. Comparing Performance in the Czech Republic, Hungary, Poland and Russia. London: Palgrave Macmillan.

Lijphart, A. (1975). "The Comparable-Cases Strategy in Comparative Research", en: Comparative Political Studies, 8 (3): 158-177.

Merton, R.K. (1957). Social Theory and Social Structure. Glencoe Ill. Free Press.

Nunberg,B.; Barbone, I. y Derlien H.U. (1999). The State after communism: Administrative transitions in Central and Eastern Europe. Washington, D.C.: World Bank.

OECD (2017). Government at a Glance. Paris : OECD http://www.oecd.org/gov/govataglance.htm.

Ongaro, E. y S. van Thiel (Eds.) (2018). The Palgrave Handbook of Public Administration and Management in Europe. Basingstoke: Palgrave Macmillan.

Otenyo, E. E. y Lind, N. S. (2006). Comparative Public Administration: the Essential Readings. Amsterdam: Elsevier JAI.

Page, E. C. y Wright, V. (1999). Bureaucratic Elites in Western European States: A Comparative Analysis of Top Officials. Oxford: Oxford University Press.

Painter, M. y Peters, B.G. (2010). "Administrative Traditions in Comparative Perspective: Families, Groups and Hybrids", en; M. Painter y B.G. Peters (Eds.) Tradition and Public Administration, Basingstoke: Palgrave Macmillan, pp. 20-30.

Patton, M. Q. (2002). Qualitative research and evaluation methods. California: Sage

Pattyn, V.; van Voorst, S.; Mastenbrock E. y Dunlop, C.A. (2018). "Policy Evaluation in Europe", en: E. Ongaro y S. van Thiel (Eds.) The Palgrave Handbook of Public Administration and Management in Europe, Vol. 1, Basingstoke: Palgrave Macmillan, pp. 577-593. 
Peters, B.G. (1988). Comparing Public Bureaucracies. Problems of Theory and Methodology, Tuscaloosa: Alabama U Press.

Peters, B.G. (2010). The Politics of Bureaucracy. An Introduction to Comparative Public Administration. London: Routledge.

Peters, B.G. (2011). Institutional Theory in Political Science. The New Institutionalism. Bloomsbury: Continuum.

Peters, B.G.; Rhodes, R.A.W. y Wright, V. (Eds.) (2000). Administering the Summit. Administration of the Core Executive in Developed Countries. Basingstoke: Macmillan,

Pierre, J. (1995). Bureaucracy in the Modern State: an Introduction to Comparative Public Administration. Cheltenham: Edward Elgar.

Pierre, J. y Peters, G.B. (Eds.) (2001). Bureaucrats and Politicians in Administrative Reform, London: Routledge

Pierson, P. (2000). "Increasing returns. Path dependence and the study of politics", in American Political Science Review, 94: 251-267.

Pollitt, C. (2011). "Not odious but onerous: Comparative public administration". Public Administration, 89(1): 114-127.

Pollitt, C. y Bouckaert, G. (2017). Public Management Reform. A Comparative Analysis into the Age of Austerity. Oxford: Oxford University Press.

Pressman, J:J. y Wildavsky, A.B. (1973). Implementation. Berkeley: University of California Press.

Przeworski, A. y Teune, H. (1970). The Logic of Comparative Social Inquiry, New York. John Wiley.

Raadschelders, J. C. N. (2011). "Commentary - Between "thick description" and large-N studies: The fragmentation of comparative research". Public Administration Review, 71(6): 831-833.

Raadschelders, J. y Vigoda-Gadot, E. (2015). Global dimensions of public administration and governance: A comparative voyage. Hoboken, NJ, USA: John Wiley \& Sons.

Riggs, F. (1962). The Ecology of Public Administration, New York. Asia Publishing.

Riggs, F. W. (1962). "Trends in the Comparative Study of Public Administration”, en International Review of Administrative Sciences, 18(1): 9-15.

Rowat, D.C. (Ed.) (1988). Public Administration in Developed Democracies, A Comparative Study. New York: Marcel Dekker

Saetren, H. y Hupe, P.L. (2018). "Policy Implementation in an Ange of Governance", en: E. Ongaro, y S. van Thiel (Eds.) The Palgrave Handbook of Public Administration and Management in Europe, vol. 1, Basingstoke: Palgrave Macmillan, pp. 553-473.

Sager, F.; Rosser, C.; Mavrot, C. y Hurni, P. (2018). A Transatlantic History of Public Administration. Cheltenham: Edward Elgar.

Salamon, L. y S. Sokolowski (2016). "Beyond nonprofits: re-conceptualizing the third sector", VOLUNTAS: International Journal of Voluntary and Nonprofit Organizations, 27(4): 1515-45.

Scharpf, F.W. (1997). Games Real Actors Play: Actor-centred Institutionalism in Policy Research. Boulder: Westview Press.

Schmidt, V. (2008). "Discursive institutionalism: the explanatory power of ideas and discourse", Annual Review of Political Science, 11(1): 303-26.

Sigelman, L. (1976). "In search of comparative administration". Public Administration Review, 36(6): $621-625$.

Suri, H. (2011). "Purposeful sampling in Qualitative Research Synthesis", en Qualitative Research Journal, 11 (2): 63-75.

UCLG (Ed.) (2008). Decentralization and local democracy in the world, First UCLG Global Report. Barcelona: UCLG.

Van de Walle, S. y Brans, Marleen (2018). "Where Comparative Administration and Comparative Policy Studies Meet”, en: Journal of Comparative Policy Analysis, 20 (1):101-113.

Van der Meer, F.M.; Raadschelders, J.C.N. y Thoonen, T.A.J. (Eds) (2007). Comparative Civil Service Systems in the 21st Century. Basingstoke: Palgrave Macmillan.

Van Dooren, W. y Hoffmann, C. (2018). "Performance Management in Europe", en: E. Ongaro y S. van Thiel (Eds.) The Palgrave Handbook of Public Administration and Management in Europe, vol. 1, Basingstoke: Palgrave Macmillan, pp. 207-221. 
Van Wart, M. R. y Cayer, N. J. (1990). “Comparative public administration: Defunct, dispersed, or redefined?", Public Administration Review, 50: 238-248, doi:10.2307/976871

Vecchi, V. y Helowell, M. (2018). "Public Private Partnerships", en: E. Ongaro and S. Van Thiel (Eds.) The Palgrave Handbook of Public Administration and Management in Europe. Vo 1 (Houndmills: Palgrave), pp. 381.402.

Verheijen, T. (Ed.) (1999). Civil Service Systems in Central and Eastern Europe. London: Edward Elgar.

Verhejen, T. y Coombes, D. (Eds.) (1998). Innovation in Public Management, Perspectives from East and West Europe. Cheltenham: Edward Elgar.

Verhoest, K.; van Thiel, S.; Bouckaert, G. y Laegreid, P. (Eds.) (2012). Government agencies. Practices and lessons from 30 countries. London: Palgrave Macmillan.

Verhoest, K.; Vandenabeele, W.; Wynen, J. y Van de Walle, S. (2018). "Challenges for large-scale international comparative survey-based research in public administration", en: E. Ongaro and S. Van Thiel (Eds.) The Palgrave Handbook of Public Administration and Management in Europe, Vol. 2 (Houndmills: Palgrave), pp. 1147-1169.

Wollmann, H. (2016). "Public and Social Services in Europe: From Public and Municipal to Private Provision - and Reverse?" en: H. Wollmann, I. Kopric y G. Marcou (Eds.) Public and Social Services in Europe. From Public and Municipal to Private Sector Provision, London: Palgrave Macmillan, pp. 313- 334.

Wollmann, H. (2018). "Public and Personal Social Services in European Countries. From Public/Municipal to Private - and Back to Municipal and "Third Sector" Provision", en International Public Management Journal, 21(3): 413-431.

Wollmann, H. (Ed.) (2003). Evaluation in Public-Sector Reform. Concepts and Practice in International Perspective. Cheltenham: Edward Elgar.

Wollmann, H. y Marcou G. (Eds.) (2010). The Provision of Public Services in Europe. Cheltenham: Edward Elgar, pp. 217.239.

Wollmann, H.; I. Kopric y G. Marcou (Eds.) (2016). Public and Social Services in Europe. From Public and Municipal to Private Sector Provision. London: Palgrave Macmillan.

\section{Notas:}

El texto fue presentado en el XXIV Congreso de la ACMS los 22-24 de noviembre, 2019 y se basa en un artículo escrito en inglés que se publicará en Oxford Encyclopedia of Public Administration, editadr Guy Peters y Ian Thynne, Oxford University Press (a aparecer).

Igualmente quiero agradecer al profesor Ángel Iglesias su ayuda en la revisión estilística al manuscrito que escribí en castellano

\section{Breve currículo:}

\section{Hellmut Wollmann}

Profesor emérito de administración pública a Humboldt Universität de Berlín, Alemania. Sus intereses de investigación y publicaciones se focalizan en los estudios comparados de políticas públicas y administración pública en países europeos. Último texto publicado: Kuhlmann, S. y Wollmann, H. (2019) Introduction to Comparative Public Administration, 2nd edition, Londres: Edward Elgar. 